\title{
Effect of phenols and phthalates on rat and human pituitary cell proliferation
}

Laura Tapella ${ }^{1}$, Antonella Sesta ${ }^{2}$, Marco Losa ${ }^{3}$, Francesco Cavagnini², Francesca Pecori Giraldi ${ }^{1,2}$

1 Department of Clinical Sciences and Community Health, University of Milan; ${ }^{2}$ Neuroendocrinology Research Lab, Istituto Auxologico Italiano; ${ }^{3}$ Department of Neurosurgery, Ospedale San Raffaele IRCCS, Milan

\section{INTRODUCTION}

There is increasing evidence, both clinical and in vitro, that pollutants affect pituitary tumor growth. Epidemiological studies have reported an increased prevalence of acromegaly and prolactin-secreting or non-functioning pituitary tumors in high industrial density areas (Cannavò 2010) and after dioxin exposure (Pesatori 2008). Several pollutants have been studied so far, in particular those proven to act as endocrine disruptors (EDs), i.e. chemicals capable of interfering with hormonal signaling. These data as well as vitro studies on endocrine disruptors suggest that environmental pollutants may affect hormonal secretion and pituitary cell proliferation (Dang 2009; Dang 2009; Dang 2007; Maruyama 1999; Elango 2006; Blake 1997; Wilson 1998).

AIM of the present STUDY was to verify the effect of endocrine disruptors on rat pituitary and human pituitary adenoma proliferative activity.

\section{METHODS}

Rat anterior pituitaries were established in culture at 50,000 cell/well density in DMEM, $10 \%$ FBS and antibiotics for 3-4 days for attachment. Cells were incubated with Benzene and bis-(2-ethylhehyl)phthalate (2-ET) at increasing concentrations (250 pM - $1.25 \mathrm{nM}$ ) for 24 and 96 hours. Control incubations were performed with plain medium only and 250 $\mu \mathrm{g} / \mathrm{ml}$ ciclohexemide (CHX). Treatments were performed in quadruplicate.

Cell viability, apoptosis and proliferation were assessed by ATP lite kit (Perkin Elmer, USA), MTT assay (Sigma, USA), Caspase 3/7 kit (Promega, USA) and 5bromo 2'-deoxyuridine labeling (BrdU labeling: Roche, Switzerland).

Five human GH-secreting pituitary adenomas, collected during transsphenoidal surgery, were established in culture and the same incubations performed. Cell viability was assessed by ATP lite kit (Perkin Elmer, USA).

Statistical comparisons were performed by non-parametric tests.

\section{Rat anterior pituitary}

Cell viability by ATP lite

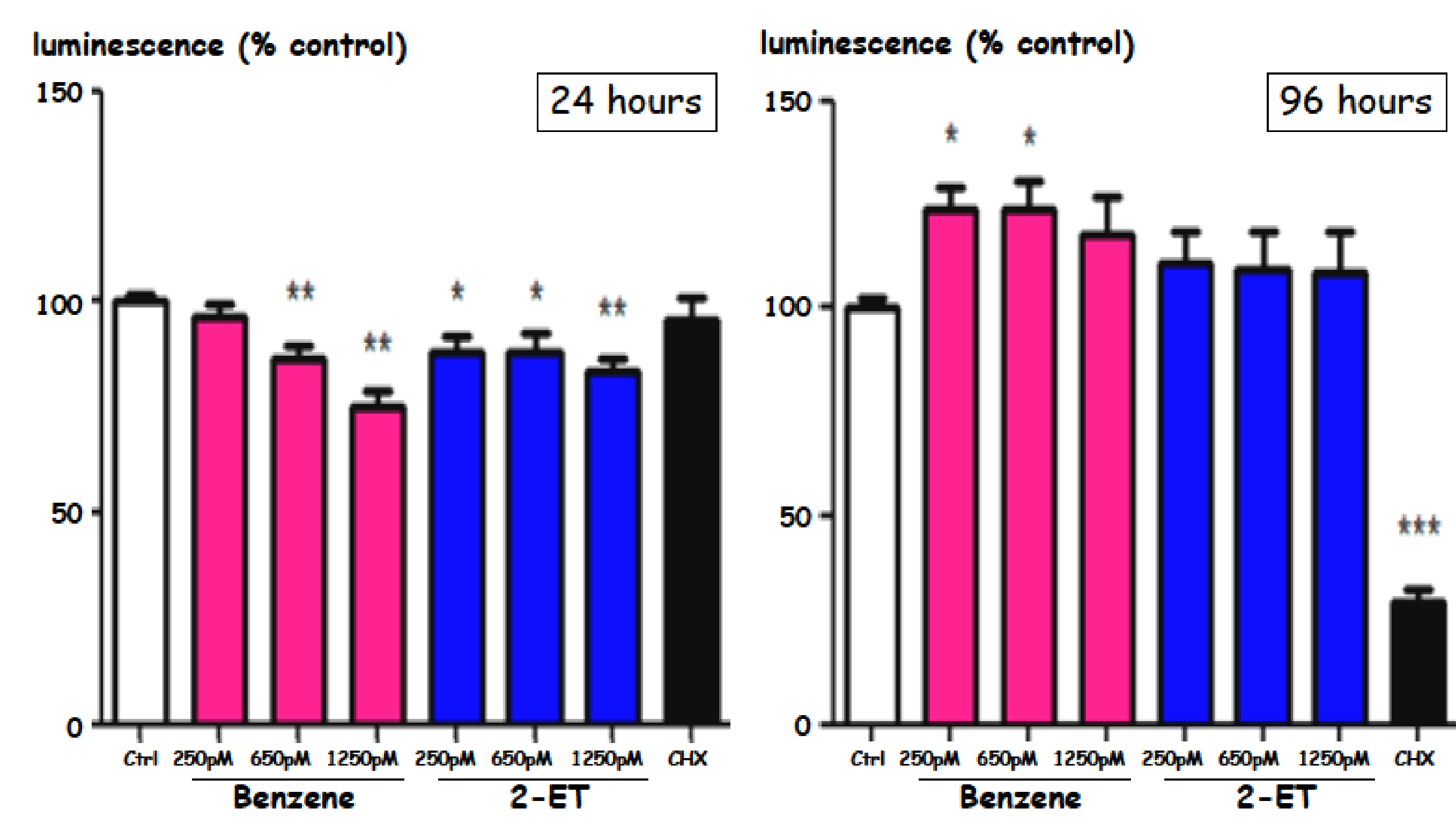

Proliferation by BrdU-labeling

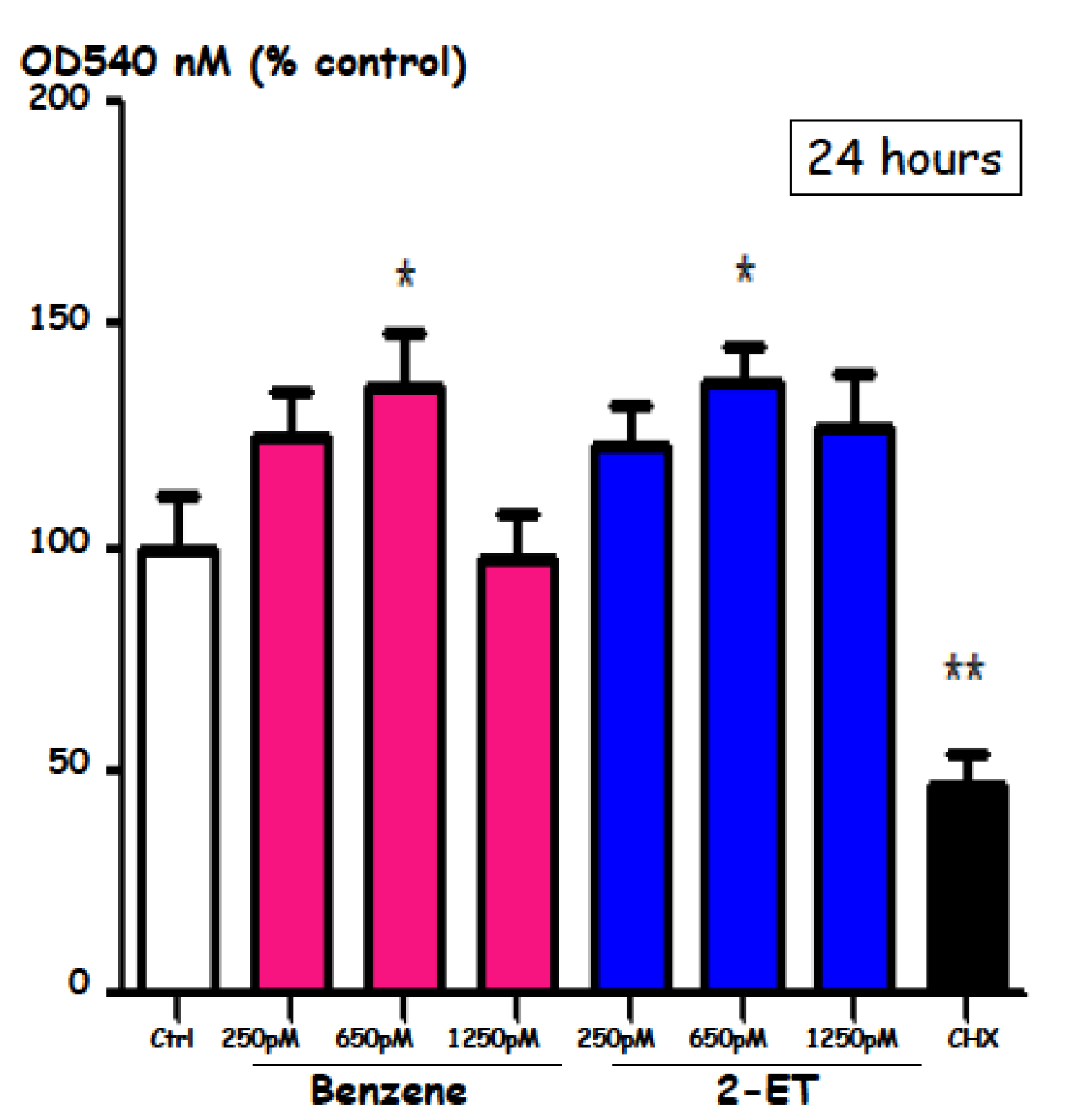

\section{RESULTS}

\section{Human GH-secreting pituitary adenomas}
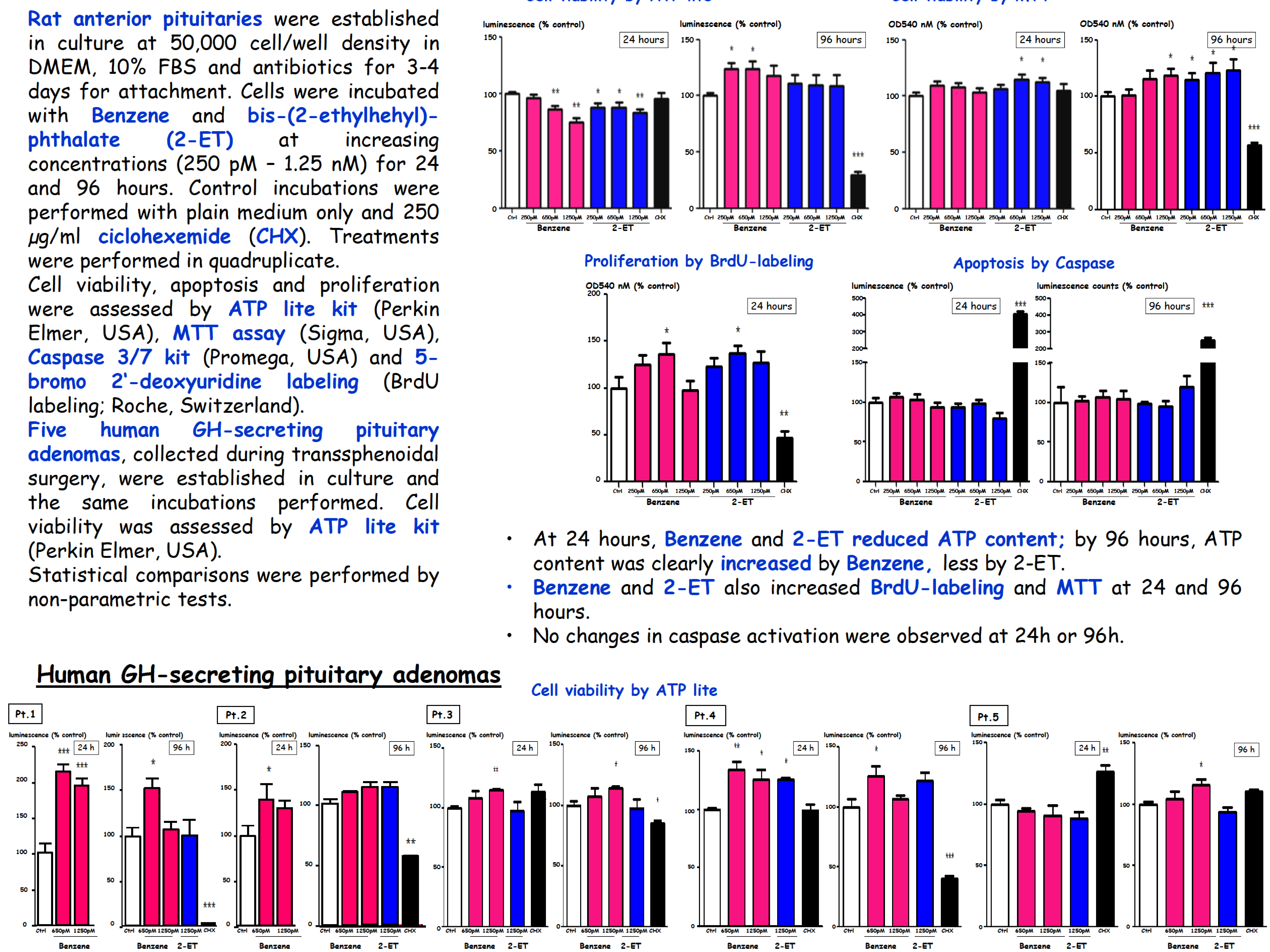

- At 24 hours, Benzene and 2-ET reduced ATP content; by 96 hours, ATP content was clearly increased by Benzene, less by $2-E T$.

- Benzene and 2-ET also increased BrdU-labeling and MTT at 24 and 96 hours.

- No changes in caspase activation were observed at $24 \mathrm{~h}$ or $96 \mathrm{~h}$.

- Benzene increased ATP content at 24 hours, less after $96 \mathrm{~h}$

- 2-ET did not affect ATP content

\section{CONCLUSIONS}

Our findings show than Benzene and bis-(2-ethylhexyl)-phthalate initially reduce then increase pituitary cell energy content; further, long-term incubation with EDs is associated with increased cell proliferation. This study indicates that both normal and adenomatous pituitary cell proliferation is modulated by endocrine disruptors, thus supporting the role of pollutants in pituitary adenoma etiology. 